\title{
Effect of random edge-vacancy disorder in zigzag graphene nanoribbons
}

\author{
J. P. C. Baldwin ${ }^{1}$ and Y. Hancock ${ }^{1,2, *}$ \\ ${ }^{1}$ Department of Physics, University of York, Heslington, York YO10 5DD, United Kingdom \\ ${ }^{2}$ York Centre for Complex Systems Analysis, University of York, Heslington, York YO10 5GE, United Kingdom
}

(Received 26 July 2016; published 12 October 2016)

\begin{abstract}
The magnetic and coherent transport properties of small-width zigzag graphene nanoribbons (ZGNRs) with monohydrogen edge passivation are investigated as a function of random edge-vacancy disorder and ribbon length. Results from noninteracting tight-binding models with (i) nearest and (ii) up to third nearest neighbor hopping are compared against those obtained from an extended mean-field Hubbard model for edge-defected ZGNRs (length $=48.02 \AA$ and width $=9.24 \AA$ ). Through ensemble averaging, a persistent magnetism and Hubbard- $U$ (i.e., spin-generated) conductance gap is found irrespective of the extent of random edge-vacancy disorder. At longer device lengths (up to $144.1 \AA$ ) and at high disorder (42.5\%), gaps open in the noninteracting model systems, whereas the gap in the Hubbard-calculated systems becomes spin dependent. In all cases, the conductance gaps increase as a function of increasing system length, although the gaps in the Hubbard systems remain smaller due to increased robustness against edge disorder. The continuance of the magnetic state and gap robustness in the ensemble-averaged Hubbard results indicates a complex interplay between the kinetics, disorder, system size, and spin interaction. Such findings may serve to reinform previous studies that have used noninteracting models to investigate disorder in ZGNRs.
\end{abstract}

DOI: 10.1103/PhysRevB.94.165126

\section{INTRODUCTION}

The structure and chemical functionalization of the edges of graphene nanoribbons (GNRs) have a pronounced effect on their spin, electronic, and transport properties [1-6]. Good quality edges at the atomic scale and controlled ribbon sizes are needed for accurate engineering of GNRs, with top-down fabrication, such as e-beam lithography, being unable to meet these aims [5,7-9]. Chemical synthesis [10,11] and unzipping carbon nanotubes $[12,13]$ may produce better quality edges. However, controlled patterning of graphene into arbitrary shapes remains within the realm (and limitations) of top-down approaches [14].

Theoretical investigations of GNRs (including patterned systems) consider mostly pristine edges, with realistic effects, such as edge disorder, being less studied. Density functional theory (DFT) simulations of disordered GNRs have been especially lacking due to the computational expense associated with large unit-cell calculations. As an example, Huang et al. probed the effect of systematic edge vacancies in small-width ZGNRs showing there to be loss of magnetism at $33 \%$ edge-vacancy concentration [15]. More realistic, yet computationally demanding, would be DFT applied to GNRs with random edge-vacancy disorder using ensemble averaging to determine the resultant properties.

Compared to DFT, model Hamiltonian calculations are amenable to investigating random disorder due to their computational efficiency and advantages in probing underlying quantum mechanisms. Previous studies using the nearest neighbor (simple) tight-binding (STB) model applied to

\footnotetext{
*y.hancock@york.ac.uk

Published by the American Physical Society under the terms of the Creative Commons Attribution 3.0 License. Further distribution of this work must maintain attribution to the author(s) and the published article's title, journal citation, and DOI.
}

GNRs have been quite extensive [16-25], with coherent transport calculations showing that random edge-vacancy disorder suppresses the conductance around the Fermi energy due to disorder-induced localization [16], or more explicitly, Anderson [26] localization [17-25]. These results have also been used to explain the conduction gap seen in experimental results [3-5].

STB calculations of ZGNRs show the ballistic transport of these systems to be more robust relative to armchair GNRs (AGNRs) against the effects of random edge-vacancy disorder [16,18-20]. Mucciolo et al. reported that the disorder-induced conduction gap in ZGNRs varies inversely as a function of the ribbon width becoming more pronounced for high edgevacancy concentrations [19]. Others have stipulated similar findings for the onset of disorder-induced gaps in ZGNRs as a function of length, width, and degree of disorder determined from STB calculations [20-22,25].

Missing from these tight-binding studies on defected ZGNRs is the effect of extended hopping and local Coulomb interaction (the so-called Hubbard- $U$ ), with the latter being essential to generate the magnetic properties and intrinsic band gap predicted by DFT [6]. In terms of an extended model, next nearest neighbor hopping reproduces the asymmetry of the ZGNR band structure, with third nearest neighbor hopping being required for the formation of band gaps in AGNRs [27] —an important consideration for ZGNRs with structural edge defects. A minimal generalized tight-binding (GTB) model capturing these features has been proposed for monohydrogen, edge-passivated GNRs and has been shown to accurately reproduce local spin density approximation DFT transport results for nontrivial, edge-defected systems [28,29].

In this work, the GTB model is applied to small-width ZGNRs to study the role of systematic versus random edgevacancy defects, and the interplay between the extended hopping, mean-field Hubbard- $U$ interaction and system size. In this respect, perturbations to the magnetism and coherent transport properties are investigated in small-width systems 
that are sensitive to defects to determine the most dominant gap-opening mechanism (i.e., Hubbard $U$ versus disorder induced).

\section{THEORY}

The GTB model is defined as

$$
H=\sum_{i \sigma} \epsilon_{i} n_{i \sigma}-\sum_{i j \sigma}\left(t_{i j} c_{i \sigma}^{\dagger} c_{j \sigma}+\text { H.c. }\right)+\sum_{i} U n_{i \sigma} n_{i-\sigma},
$$

where $c_{i \sigma}^{\dagger}\left(c_{j \sigma}\right)$ is the fermion creation (destruction) operator, which creates (destroys) an electron with spin $\sigma=\{\uparrow, \downarrow\}$ at site $i(j)$, and $n_{i \sigma}=1$ or 0 is the spin-dependent number operator. H.c. is the Hermitian conjugate. To model the edge-vacancydefected ZGNRs, the parameter set in Hancock et al. (2010) is used with on-site energy $\epsilon_{i}$ equal to zero, and the Hubbard- $U$ (the local Coulomb interaction energy between opposite spins) [30] set to $2.0 \mathrm{eV}$. The hopping energies $t_{i j}$ from this parameter set are $2.7 \mathrm{eV}, 0.20 \mathrm{eV}$, and $0.18 \mathrm{eV}$ for first, second, and third nearest neighbor hopping, respectively. These terms describe the spin kinetics of the system. In addition to the STB and GTB models, an ETB (extended tight-binding model) is defined consisting of the extended hopping terms only (up to third nearest neighbor).

The GTB model is solved within the mean-field approximation for a half-filled system, where

$$
n_{i \sigma} n_{i-\sigma}=\left\langle n_{i \sigma}\right\rangle n_{i-\sigma}+n_{i \sigma}\left\langle n_{i-\sigma}\right\rangle-\left\langle n_{i \sigma}\right\rangle\left\langle n_{i-\sigma}\right\rangle,
$$

and $\left\langle n_{i \sigma}\right\rangle$ is the expectation associated with the local spin occupancy. Using this approximation, Eq. (1) is decoupled and linerarized into two spin-dependent Hamiltonians, which are solved self-consistently. For more specifics about this method, see Hancock and Smith (2003) [31].

Vacancy-defected ZGNRs are modeled either by systematic or random removal of edge atoms. When randomly removed, the edge atoms are equally weighted and then selected using a pseudorandom number generator [32]. The removal process then continues until the required edge-vacancy concentration has been achieved. Examples of random edge-defected ZGNRs used in this study are shown in Fig. 1. Klein defects, which are difficult to model due to the experimentally observed vibration of the $\mathrm{C}-\mathrm{C}$ bond [33], have been removed by defining the edge as the first two atomic rows of carbon atoms (Fig. 2). A 20-5-ZGNR (Fig. 1) therefore has 40 carbon atoms per edge, with the percentage concentration of random edge-vacancy defects calculated over both edges.

The magnetic properties of the ZGNRs are determined by calculating the local spin polarization

$$
p_{i}=\frac{\left\langle n_{i \uparrow}\right\rangle-\left\langle n_{i \downarrow}\right\rangle}{\left\langle n_{i \uparrow}\right\rangle+\left\langle n_{i \downarrow}\right\rangle} .
$$

Averaged over the entire device,

$$
p_{\text {total }}=\frac{\sum_{i} p_{i}}{N}
$$

is the net average spin polarization per atom, where $N$ is the total number of atoms.

The coherent transport properties of the ZGNRs are modeled using the Landauer-Büttiker formalism [34] assuming the device has ideal, semi-infinite ZGNR leads. The

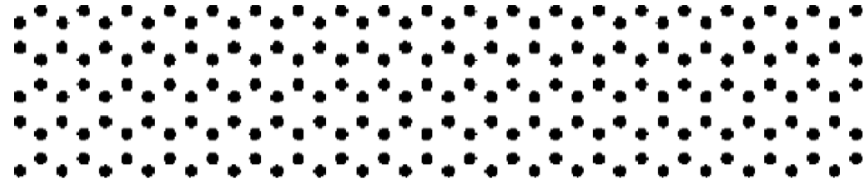

(a)

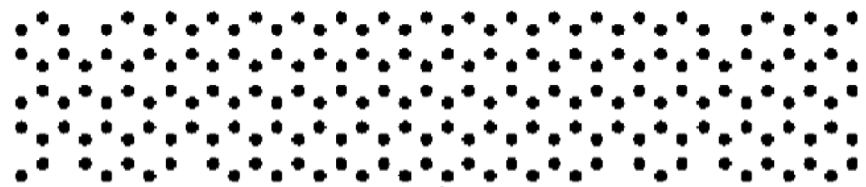

(b)

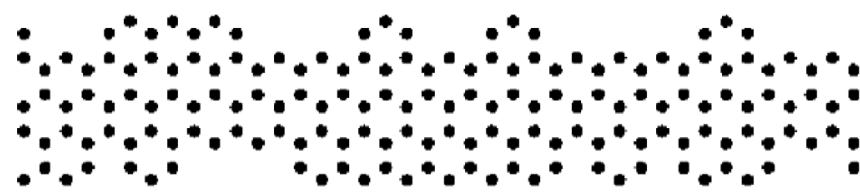

(c)

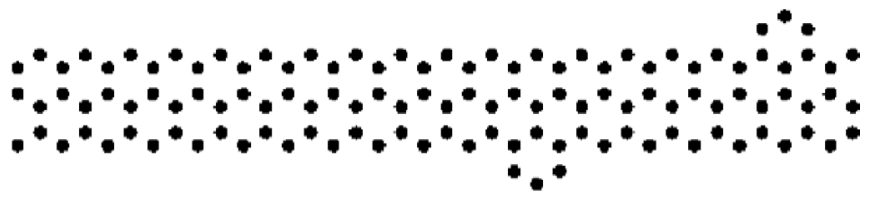

(d)

FIG. 1. (a) Ideal 20-5-ZGNR device used in this work that is 20 carbon atoms long (48.02 $\AA$ ) and 5 carbon atoms wide $(9.24 \AA)$. 20-5-ZGNRs with randomly-generated (b) $7.5 \%$, (c) $42.5 \%$, and (d) $90 \%$ edge-vacancy concentrations.

spin-dependent conductance $G_{\sigma}(E)$ is obtained from the transmission function $T_{\sigma}(E)$ such that

$$
G_{\sigma}(E)=\frac{e^{2}}{h} T_{\sigma}(E),
$$

where $E$ is the energy, and $e$ and $h$ are the electron charge and Planck's constant, respectively. Here,

$$
T_{\sigma}(E)=\operatorname{Tr}\left[\Gamma_{L \sigma}(E) G_{\sigma}^{\mathrm{Ret}}(E) \Gamma_{R \sigma}(E) G_{\sigma}^{\mathrm{Adv}}(E)\right],
$$

where $G_{\sigma}^{\text {Ret/Adv }}(E)$ are the retarded/advanced Green's functions. The left $(L)$ and right $(R)$ lead gamma functions $(\Gamma)$ are obtained from the difference in the retarded $\Sigma_{L / R \sigma}^{\text {Ret }}(E)$ and

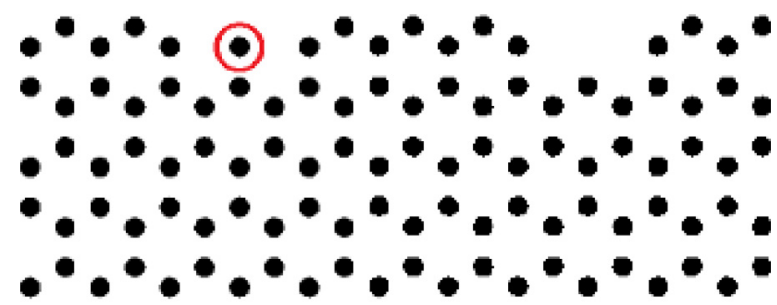

(a)

(b)

FIG. 2. Schematic of an edge-vacancy-defected portion of a 205-ZGNR showing (a) a Klein defect (circled), and (b) a notch-defect extending into the second atomic row after the Klein defect has been removed. 


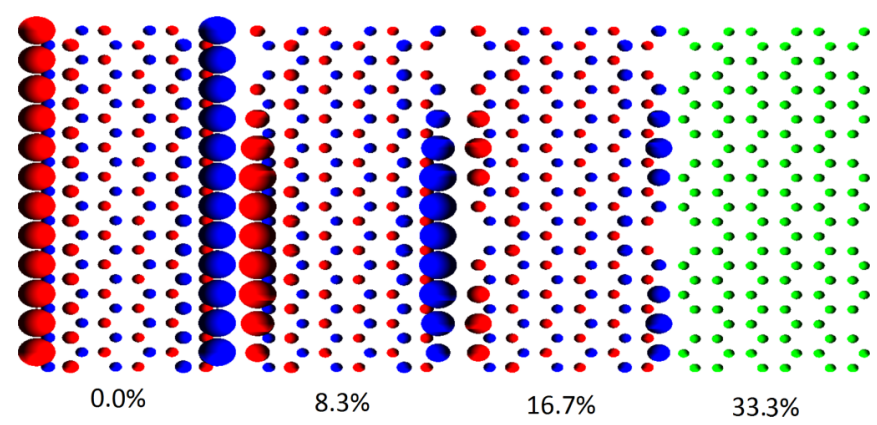

FIG. 3. GTB results of the local net spin polarization for the systematic edge-vacancy-defected ZGNRs studied by Huang et al. [15]. Blue circles are net spin-up and red circles are net spin-down. Green circles are net zero spin polarization. The edge-vacancy concentrations are $0.00,0.034,0.068$, and $0.136 \AA^{-1}$ from left to right (Huang et al.'s convention) with equivalent percentage edge-vacancy concentrations also shown.

advanced $\Sigma_{L / R \sigma}^{\mathrm{Adv}}(E)$ self-energies of the leads, such that

$$
\Gamma_{L / R \sigma}(E)=\left[\Sigma_{L / R \sigma}^{\mathrm{Ret}}(E)-\Sigma_{L / R \sigma}^{\mathrm{Adv}}(E)\right]
$$

and

$$
\Sigma_{L / R \sigma}^{\operatorname{Ret} / \operatorname{Adv}}(E)=V_{L / R}^{\dagger} g_{L / R \sigma}^{\operatorname{Ret} / \operatorname{Adv}}(E) V_{L / R},
$$

where $V_{L / R}$ is the coupling between the ZGNR device and the L/R lead. The retarded/advanced surface Green's functions for the leads $g_{L / R \sigma}^{\text {Ret/Adv }}(E)$ are obtained using the decimation iteration method [35] and the transmission function [Eq. (6)] is calculated using the methods described in Ref. [36]. A grid spacing in $E$ of $0.008 \mathrm{eV}$ is used in these calculations.

\section{RESULTS AND DISCUSSION}

A. ZGNRs with systematic edge vacancies

The GTB model is first tested by applying it to the systematically edge-defected ZGNRs previously investigated by Huang et al. using local spin density approximation DFT. These systems have length $L=28.3 \AA$, width $W=$ $11.4 \AA$, and a defected region defined as the first row of atoms along the top and bottom edges of the ribbon [15]. Using Huang et al.'s convention, the edge-vacancy concentrations per unit cell length are $0.00,0.034,0.068$, and $0.136 \AA^{-1}$, or equivalently, $0 \%, 8.3 \%, 16.7 \%$, and $33.3 \%$.

The GTB results for the local spin polarization per atom $\left(p_{i}\right)$ as a function of the edge-vacancy concentration are calculated within the unit-cell approximation (Fig. 3). The radii of the circles are the local net spin polarizations scaled by $0.5+5^{*} p_{i}$ to allow for easy visualization. The GTB spin distributions show the local net spin polarization decreases with increasing edge-vacancies concentration and that the system becomes paramagnetic at $33.3 \%\left(0.136 \AA^{-1}\right)$ edgevacancy concentration, agreeing with Huang et al.'s DFT prediction [15].

Although a seemingly interesting result, the loss of magnetism in the $33.3 \%$ systematically edge-defected Huang et al. system is found to be an artefact of finite size effects and the specific choice of systematic edge disorder. Probing finite size

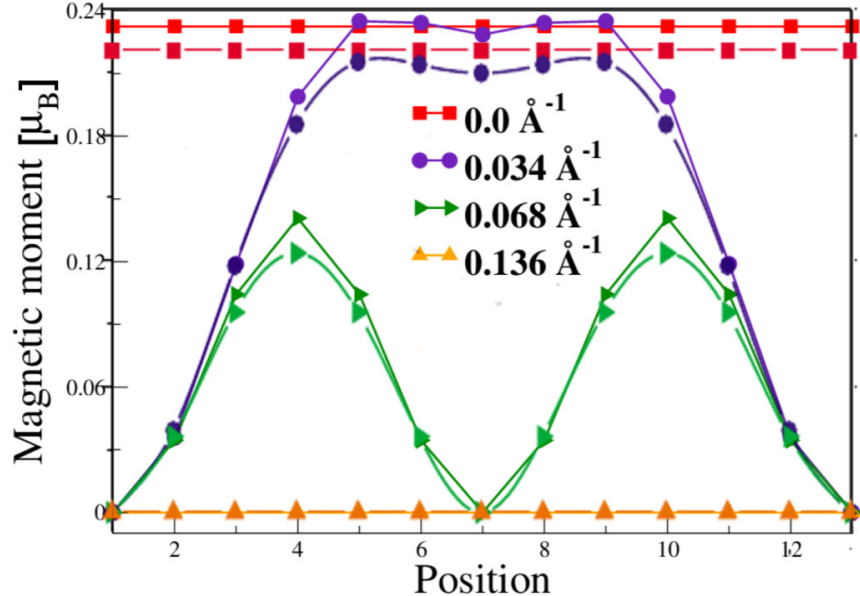

(a)

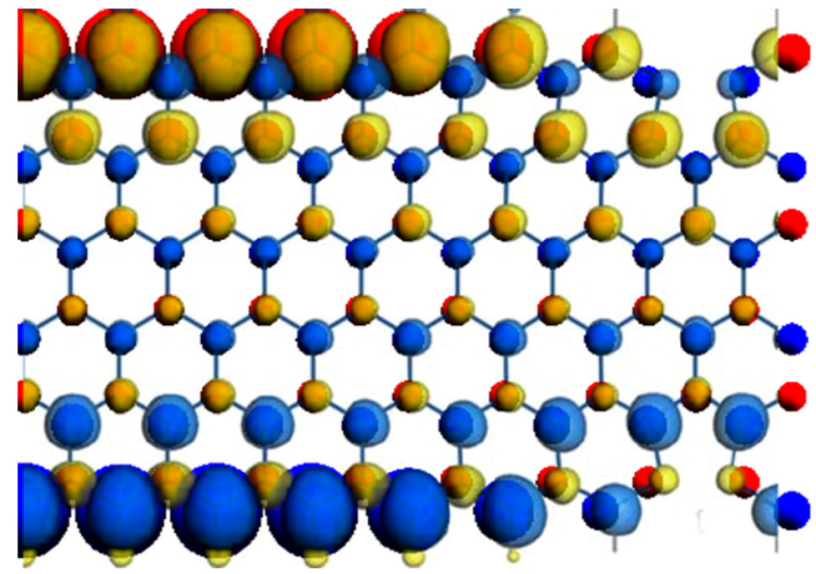

(b)

FIG. 4. (a) Quantitative values taken from the GTB results (Fig. 3) of the local magnetic moment as a function of the atomic-edge position superimposed over Huang et al.'s results. (b) Example of the structural and magnetic differences between the GTB and Huang et al. systems. Images from Huang et al. used with permission.

scaling using the GTB model by doubling the unit cell size for the $33.3 \%$ edge-vacancy-defected Huang et al. system reveals numerous structural configurations where the magnetism is not quenched. Increasing the unit cell size and, in turn, increasing the length of the ribbon therefore makes it possible for magnetism to be maintained in edge-vacancy-defected monohydrogen-passivated ZGNRs due to regions of extended, preserved ZGNR edge.

A site comparison of the local spin polarization per edge atom (magnetic moment) as a function of the percentage edge-vacancy concentration between Huang et al.'s DFT and the GTB results shows good agreement [Fig. 4(a)]. Small differences between the GTB and DFT solutions are attributed to the ionic relaxation in the DFT calculation, which is not fully accounted for in the minimal GTB model [Fig. 4(b)]. The largest structural differences are around the defect site [Fig. 4(b)] consistent with the GTB model being parameterized against fully-relaxed SIESTA results for ideal GNRs [28]. Given the good agreement shown in Fig. 4(a), and with the view of maintaining a minimal model approach, we conclude that the GTB model adequately describes the magnetism in 


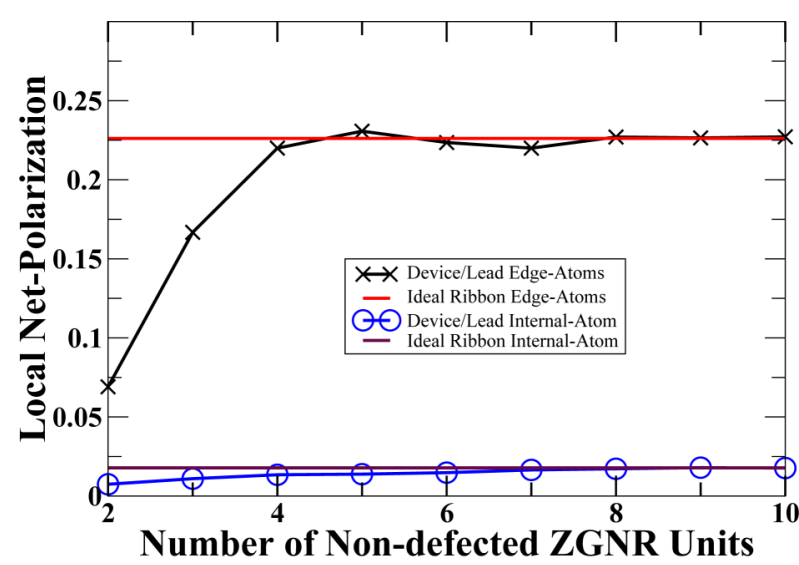

(a)

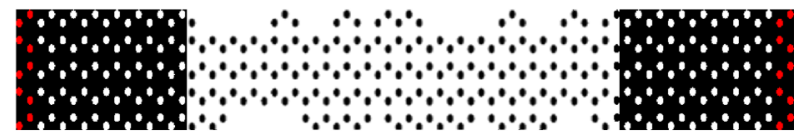

(b)

FIG. 5. (a) The local net spin polarization at the lead-device interface on an edge atom $(\mathrm{x})$ and internal atom (o) for a $51.3 \%$ edgevacancy-defected 20-5-ZGNR device as a function of the number of ideal ZGNR units in the lead region. Convergence against the ideal system results (horizontal lines) occurs at a nondefected lead length of eight 5-ZGNRs. (b) Structure of the defected 20-5-ZGNR device with the required eight 5-ZGNR-unit ideal leads (black background).

edge-defected ZGNRs and move forward to study the effect of random edge vacancies in small-width systems.

\section{B. Random edge-vacancy-defected ZGNRs}

In this section, the effect of random edge-vacancy defects are studied in 20-5-ZGNRs (Fig. 1) with their properties calculated using ensemble averaging. The small size of these systems enables a good range of edge-vacancy concentrations to be probed while remaining computationally tractable with respect to the total number of atoms and Hubbard- $U$ convergence. The reduced width of the systems also means that they are sensitive to defect-induced transport perturbations and to changes in size arising from the kinetics and Coulomb interactions in the model.

\section{Convergence tests and ensemble averaging}

Before calculating the transport properties of the randomly defected 20-5-ZGNRs, a convergence test is required to ensure continuity of the magnetism from the device into the semi-infinite ideal ZGNR leads. To perform this test, the local spin polarization on the edge and internal atoms of a $51.3 \%$ heavily-defected 20-5-ZGNR is measured as a function of the number of ideal ZGNR units in the lead region [Fig. 5(a)]. The result shows that a minimum length of eight 5-ZGNR units is required before the magnetism at the end of the leads matches that of an ideal 5-ZGNR (this being the convergence criterion). This additional lead length [Fig. 5(b)] is then used in all subsequent transport calculations.

To determine the effect of random edge vacancies on the calculated properties of the ZGNRs, an ensemble average is

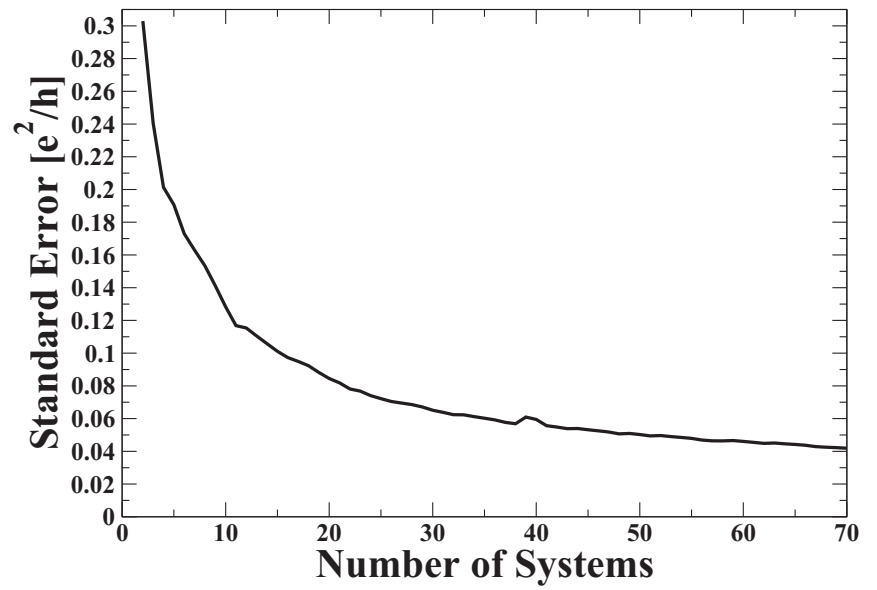

FIG. 6. Convergence of the maximum standard error associated with the ensemble-averaged spin-dependent GTB conductance determined in the range $1.5 \mathrm{eV} \leqslant E-E_{F} \leqslant 1.5 \mathrm{eV}$ for a $7.5 \%$ random edge-vacancy-defected 20-5-ZGNR.

taken:

$$
\chi \approx \bar{x}=\frac{1}{N} \sum_{i=1}^{N} x_{i},
$$

where $x_{i}$ is the measured property for the $i$ th system in a sample size of $N, \bar{x}$ is the average value of that property taken over $N$, and $\chi$ is the ensemble average. Each system in the ensemble average is equally weighted as the variation in the total energy per system is small (approximately $\pm 10^{-4} \mathrm{eV}$ ).

The confidence in the sample mean of the measured property is calculated using the standard error:

$$
S E=\frac{\sigma}{\sqrt{N}},
$$

where $\sigma$ is the standard deviation. To determine a representative value of $N$, a convergence test is performed for the maximum $S E$ pertaining to the ensemble-averaged spindependent GTB conductance of a 7.5\% edge-vacancy-defected 20-5-ZGNR in the range $1.5 \mathrm{eV} \leqslant E-E_{F} \leqslant 1.5 \mathrm{eV}$ (Fig. 6) (this being a key property investigated in this work). A 7.5\% defected system is selected as this leads to a maximum number of $N$ possible system configurations. The results for the convergence test show that a minimum of $N=50$ configurations is adequate to ensure sufficient maximum statistical accuracy of $\pm 0.06 e^{2} / h$ while still allowing for the GTB calculations to remain computationally tractable.

\section{Ensemble-averaged magnetic properties}

The antiferromagnetic, lowest-energy state in monohydrogen edge-passivated ZGNRs (Z1-edge) is attributed to Leib's theorem based on the Hubbard model applied to a bipartite lattice [37]. More recently, Clar's aromaticity rule [38,39] has provided a simple means of predicting and explaining magnetism in GNRs such that nonaromatic edges with $<1 / 3$ aromaticity are magnetic [40,41]. In this respect, an ideal Z1-edge, which is $100 \%$ nonaromatic can have aromaticity introduced by adding vacancy-formed, monohydrogen passivated armchair edge structure. 


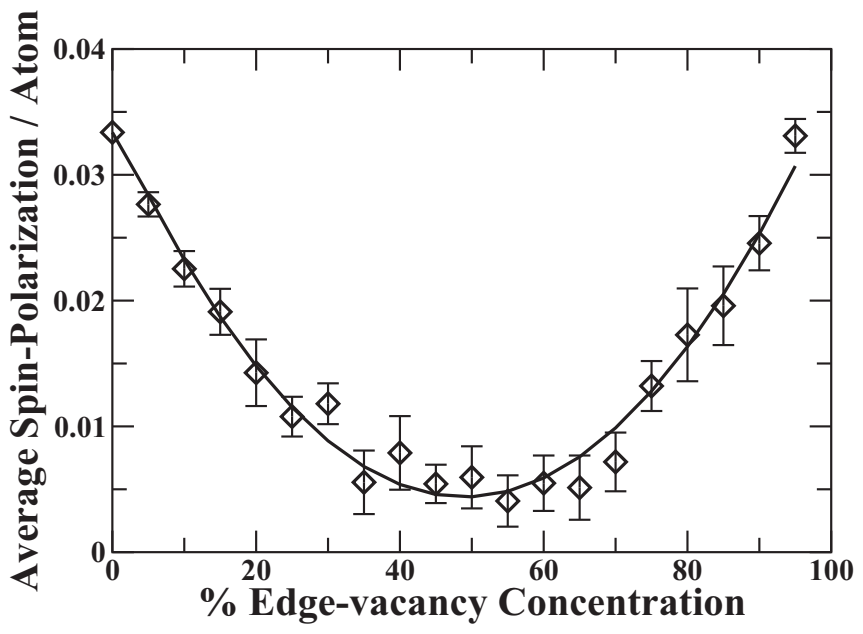

FIG. 7. GTB prediction for the ensemble-averaged net spin polarization per atom in 20-5-ZGNRs showing persistent magnetism throughout the edge-vacancy concentration range. Error bars correspond to the standard errors associated with these measurements. A parabolic fit to these data is included to guide the eye.

Against the ideal Z1 result, the edge-vacancy-defected 205-ZGNRs have a parabolic variation in the ensemble-averaged net spin polarization per atom as a function of the increasing percentage of random edge-vacancy defects (Fig. 7). At each edge-vacancy concentration, the ensemble-averaged result remains antiferromagnetic in contrast to the systematic edgedefected 12-6-ZGNR, which became paramagnetic at $33.3 \%$ edge-defect concentration (cf. Fig. 3). With respect to Clar's aromaticity rule, the persistent magnetism in the 20-5-ZGNR can be explained by a finite probability of systems in the ensemble population having nonaromatic magnetic edges. This understanding also explains how doubling the unit cell length for the $33.3 \%$ edge-vacancy-defected Huang et al. system reveals several configurations where magnetism is preserved due to local regions of nondefected ZGNR edge (see also the previous discussion in Sec. A).

The possibility of persistent magnetism in random edgevacancy-defected GNRs provides support for the use of ensemble averaging and an interacting spin-dependent model, such as the GTB, in preference to noninteracting STB and ETB models. This brings to bear an important consideration as to how the local Coulomb interaction (i.e., Hubbard term), in conjunction with other mechanisms, such as disorderinduced localization, determines the coherent transport and gap-forming properties of these systems.

\section{Ensemble-averaged transport}

To answer this question, we investigate the key gapformation processes arising from the extended hopping and mean-field Hubbard- $U$ in random edge-vacancy-defected 205-ZGNRs by comparing the ensemble-averaged STB, ETB, and GTB coherent transport results as a function of the edgevacancy concentration. For detailed transport comparisons, representative edge-vacancy concentrations of $7.5 \%, 42.5 \%$, and $90 \%$ (low to high) are chosen (see Fig. 1).
Irrespective of the model used and degree of edge-vacancy concentration, edge-vacancy defects reduce the overall chargecarrier conduction compared to the ideal (nondefected) results in agreement with other STB studies [16,19,21,23] (Fig. 8). At low (7.5\%) edge-vacancy concentration, both the STB and ETB noninteracting systems remain metallic about the Fermi energy $\left(E_{F}\right)$ with the ETB result exhibiting a greater conductance due to increased kinetics caused by the extended hopping [Fig. 8(a)]. In comparison, the GTB result shows the width of the Hubbard- $U$ gap remains relatively impervious to disorder despite the overall reduced conductance caused by the defect-induced removal of conduction channels [inset of Fig. 8(a)]. Both observations can be explained by the robustness of the edge states against disorder in the noninteracting and interacting systems.

At increased edge-vacancy concentration (42.5\%), a significant reduction in the overall conductance occurs for the defected systems bringing the STB, ETB, and GTB results into closer alignment [Fig. 8(b)]. Closer inspection of the gap region (inset) shows that the width of the GTB gap remains intact despite an even greater reduction in the overall conductance. Due to larger notched regions and pronounced structural asymmetry across the ribbon edges, the GTB result has also become spin dependent. In comparison, the STB conductance is significantly reduced in the gap region with large gaps induced due to a substantial loss of conduction channels. In the ETB result, which has increased kinetics, there is still notable conduction in the gap region with indication of conduction dips, again due to the removal of numerous conductance channels. In both the STB and ETB cases, the conduction at $E_{F}$ persists against the measured local maximum $S E$ uncertainty of $\pm 0.008 e^{2} / h$.

With a further increase in the edge-vacancy concentration to $90 \%$, the ensemble-averaged STB, ETB, and GTB conductance results appear to recover [Fig. 8(c)]. This recovery is particularly apparent in the gap region for the STB and ETB results and is associated with these systems becoming more similar to a smaller-width 20-3-ZGNR device with $10 \%$ added edge-atom concentration [cf. Fig. 1(d)]. In this respect, the structural protrusions that have caused significant loss of conductance, hence increased charge-carrier localization, have been progressively removed as the edge-vacancy concentration increases above $50 \%$.

A quantitative comparison of the degree of conductance in these systems is made by plotting the integrated conductance calculated via Simpson's rule over the range $-0.5 \mathrm{eV}<$ $E-E_{F}<0.5 \mathrm{eV}$ as a function of increasing edge-vacancy concentration [Fig. 9(a)]. The results show similar trends in the integrated conductance across all three models with the greatest measured differences occurring at $0-20 \%$ and 70-100\% edge-vacancy concentrations. Within these ranges, the STB results tend toward the GTB results, whereas the ETB shows, in general, greater conductance due to increased kinetic degrees of freedom. Between $32.5 \%$ and $51.3 \%$ edge-vacancy concentrations, all three models closely coincide as each system becomes similarly affected by the loss of conduction channels and the increase in notched edge structure.

A detailed study of the conduction measured at $E_{F}$ as a function of the edge-vacancy concentration shows persistence of the metallic state in the STB and ETB systems [Fig. 9(b)]. 


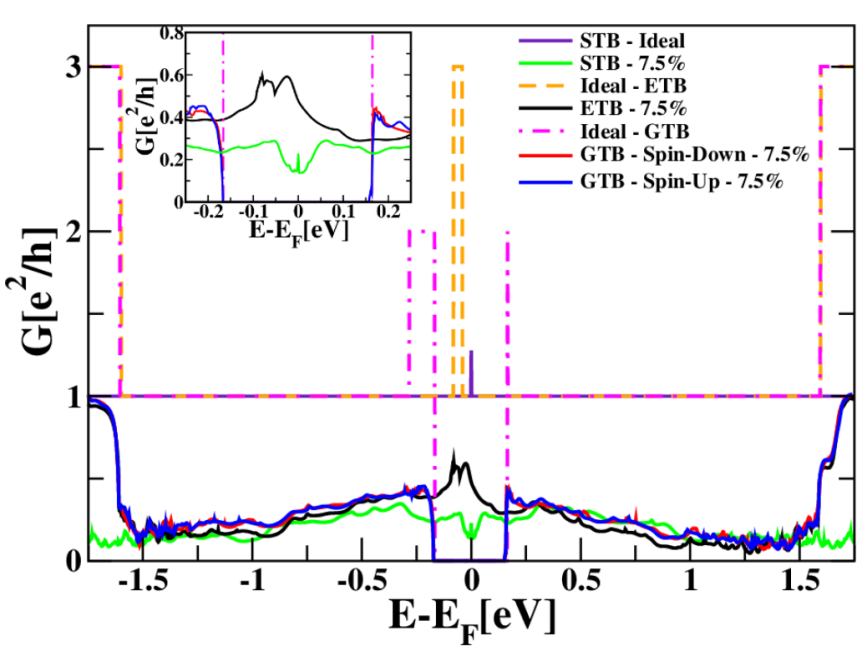

(a)

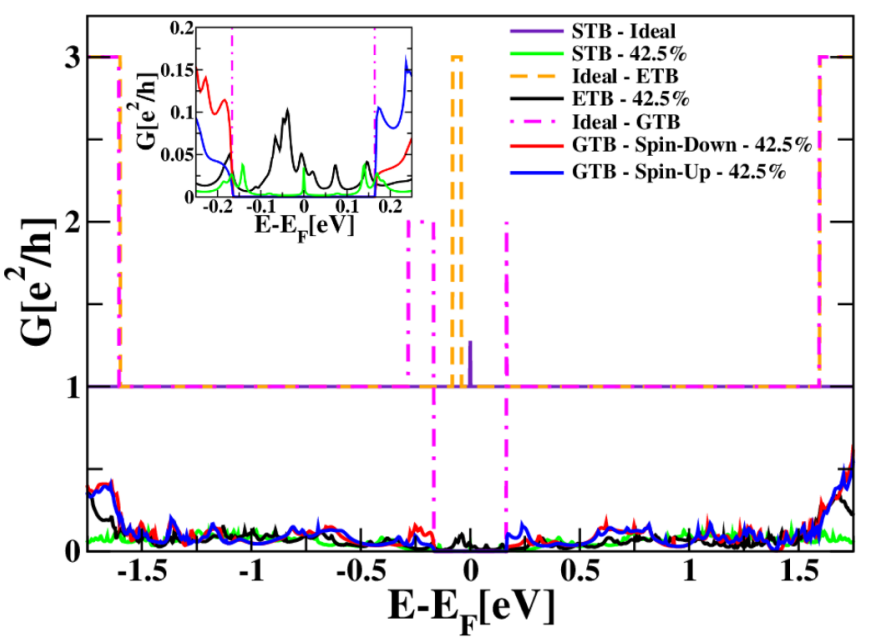

(b)

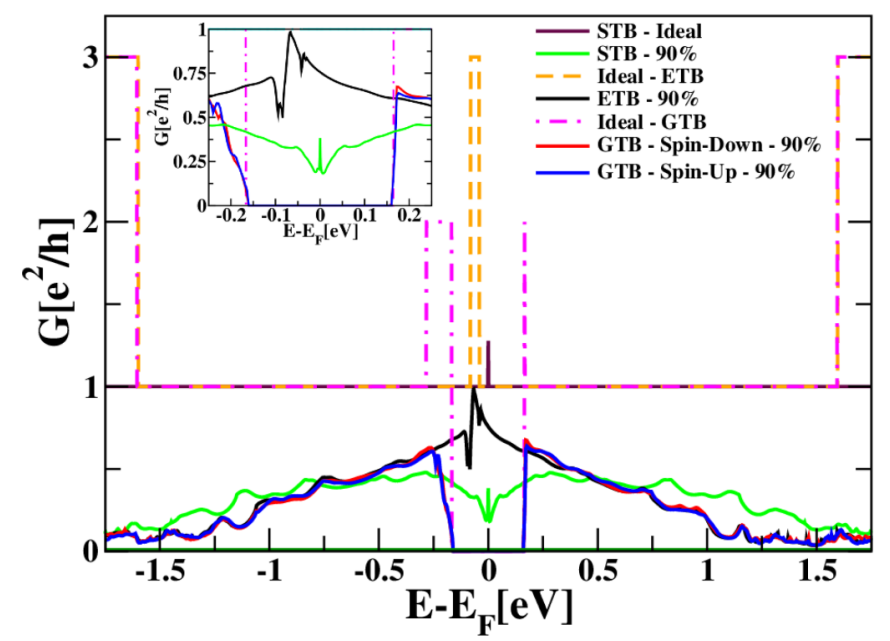

(c)

FIG. 8. STB, ETB, and GTB results for the ensemble-averaged conductance $(G)$ as a function of the energy $E$, relative to the Fermi energy $E_{F}$, in an ideal 20-5-ZGNR, and with (a) $7.5 \%$, (b) $42.5 \%$, and (c) $90 \%$ random edge-vacancy concentrations. The insets are zoomedin views of the conductance about $E_{F}$. A maximum uncertainty of $\pm 0.06 e^{2} / h$ occurs in the $S E$ of these results. However, in the $42.5 \%$ noninteracting solutions, a $\pm 0.008 e^{2} / h$ local $S E$ uncertainty occurs at $E_{F}$, thus maintaining finite conductance at this point.

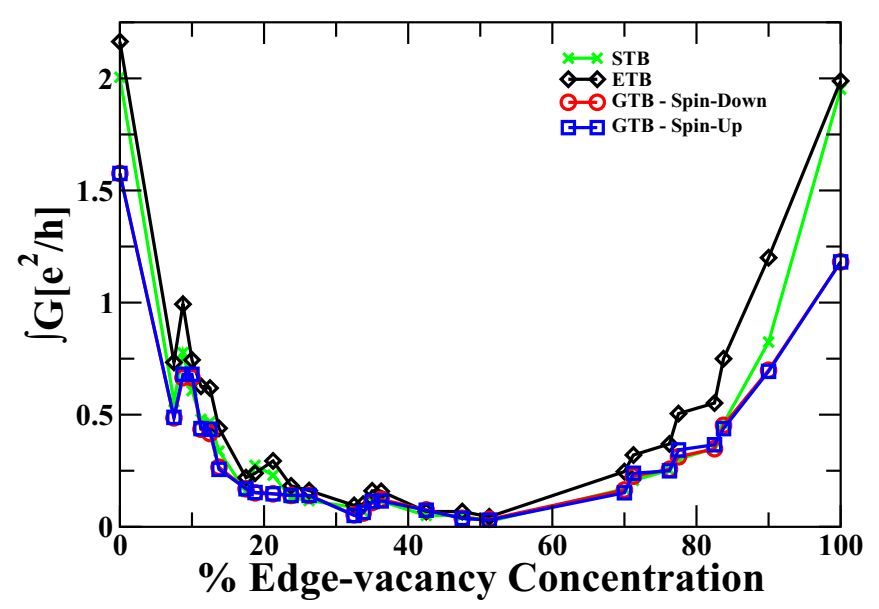

(a)

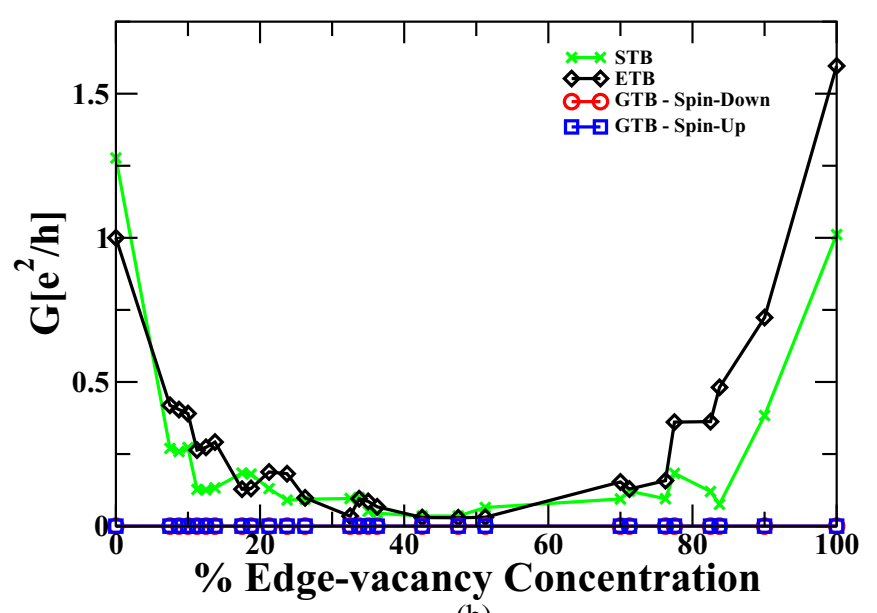

(b)

FIG. 9. (a) The integrated STB, ETB, and GTB ensembleaveraged conductance $\left(\int G\right)$ measured over the energy range $-0.5 \mathrm{eV}<E-E_{F}<0.5 \mathrm{eV}$, and (b) comparison of the ensembleaveraged conductance measured at $E_{F}$ for the STB-, ETB-, and GTBcalculated 20-5-ZGNRs, as a function of increasing edge-vacancy concentration. The conductance at $E_{F}$ in the noninteracting models does not go to zero despite accounting for the $\pm S E$ uncertainties of these measurements.

In the GTB system, the conductance at $E_{F}$ remains zero due to the intrinsic conduction gap. Although the results for all three models very closely agree between $42.5 \%$ and $51.3 \%$ edgevacancy concentrations, the values for the conductance do not extend below the criterion for equivalent zero conductance (i.e., less than $0.01 * e^{2} / h$ for single spin $[5,20,21]$ ), even when taking into account the maximum uncertainty in the standard error of these measurements $\left( \pm 0.008 e^{2} / h\right.$ in this vacancy concentration range). Thus, at no point as a function of the edge-vacancy concentration are the STB and ETB results zero conducting at $E_{F}$.

We conclude that noninteracting models, such as the widely-applied STB model and the ETB model, are not able to describe the conduction properties of these systems due to the GTB predictions of (i) finite magnetism, (ii) spin-dependent transport, and (iii) an intrinsic conduction gap. The Hubbard- $U$ remains the chief mechanism for conduction gap formation irrespective of the edge-vacancy concentration due to the 
resilience of the STB edge-state and increased kinetics in the ETB systems. The stability of the GTB conductance gap and system magnetization, even for highly edge-disordered devices (for example, at $42.5 \%$ edge-vacancy concentration), means that the Coulomb interactions in these systems cannot be ignored. To investigate these effects further, the localization properties for the edge-vacancy-defected 20-5-ZGNR will be studied using the STB, ETB, and GTB models, and subsequent to this, the localization properties will also be determined as a function of the device length.

\section{Localization studies}

At a given energy $E$, the charge-carrier localization length $\zeta$ is related to the conductance due to disorder $G^{\prime}$ and the conductance of the ideal system $G$ via

$$
G^{\prime}(L)=G(L) e^{\frac{-L}{\zeta}},
$$

where $L$ is the length of the device [42-45]. Therefore, at constant $L$, the localization length is

$$
\zeta=\left(-\ln \left[G / G^{\prime}\right] / L\right)^{-1},
$$

for a single point measurement (see Ref. [16] for the use of this equation in the study of GNRs). For systems studied as a function of $L$, the localization length $\zeta$ is determined from the inverse of the slope of the $-\ln \left[G(L) / G^{\prime}(L)\right]$ versus $L$ curve. In both cases, charge-carrier localization is said to occur when $\zeta$ is smaller than the device length $L$. As a first approximation, we will calculate the localization length for the single-width 20-5-ZGNR, and then move to a more accurate determination of the localization regime using Eq. (11) as a function of increasing device length.

The exponential decay of the conductance as a function of device length [Eq. (11)] has been established in GNRs using the STB model $[16,19,21,23]$. In these studies, ZGNRs were found to be significantly more robust compared to AGNRs against random edge-vacancy perturbations [16]. The fact that the localization length is inversely dependent on ribbon width $[16,19,21,23]$ coupled with the insensitivity of ZGNRs to edge perturbation at large widths $[16,21]$ provides additional supporting evidence for the choice of ultra-thin ZGNRs in this work.

Using Eq. 12, the ensemble-averaged localization length $\zeta$ is calculated for the 20-5-ZGNR at $7.5 \%$ and $42.5 \%$ edge-vacancy concentrations (Fig. 10). The results show a significant increase in charge-carrier localization as indicated by the reduced localization length in the device at increasing edge-vacancy concentration $(7.5 \%$ to $42.5 \%)$ independent of the model used. The increase in charge-carrier localization coincides with the decrease in overall conductance in Fig. 9. Note that the $90 \%$ random edge-vacancy concentrated system was not included here due to the increase in structurally ideal edge in this system leading to a similar effect on the conductance as the $7.5 \%$ result [Fig. 10(a)].

These first approximation results for the 20-5-ZGNR device ( $L=48.02 \AA$ ) show it to be within the localization regime at all energies at $42.5 \%$ edge-vacancy concentration, independent of the model used [Fig. 10(b)]. At 7.5\% edge-vacancy concentration, only the STB result is within the localization regime at all energies investigated [Fig. 10(a)]. The onset

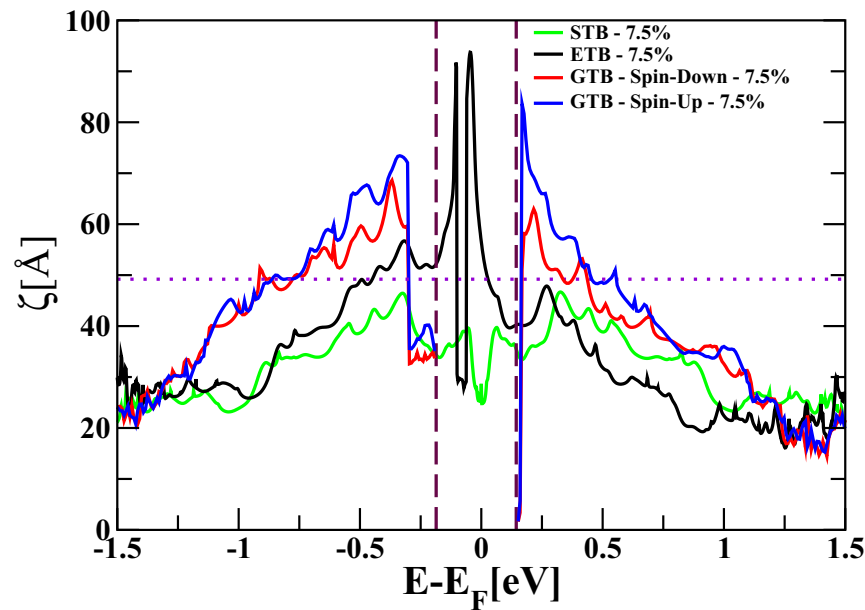

(a)

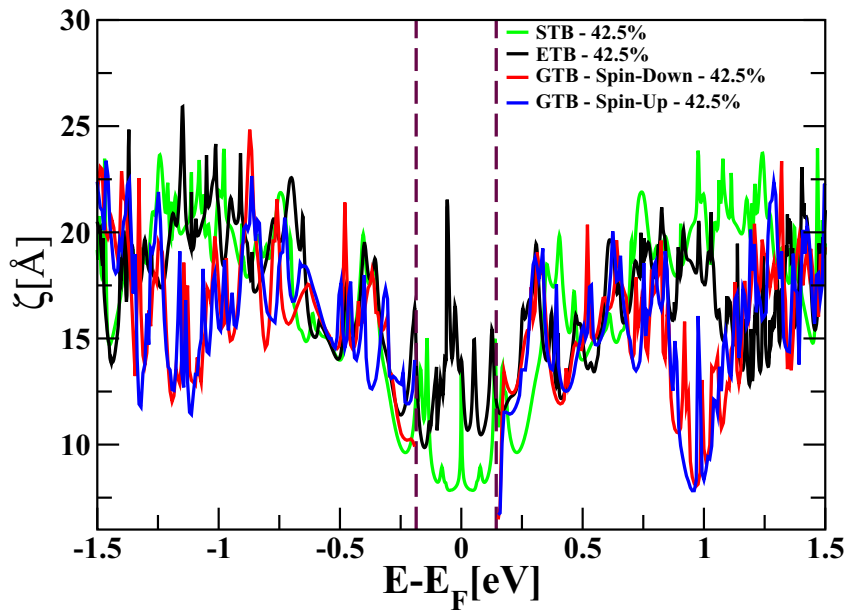

(b)

FIG. 10. Effect of increasing the edge-vacancy concentration on the ensemble-averaged localization length $(\zeta$ ) calculated for a 20-5ZGNR with (a) $7.5 \%$ and (b) $42.5 \%$ edge-vacancy concentrations. The Hubbard- $U$ gap for an ideal GTB system is indicated by the vertical dashed lines. In (a), a horizontal dotted line has been included to signify the device length of $48.02 \AA$. The maximum $S E$ uncertainty associated with the localization lengths in the ensemble average is $\pm 3 \AA$.

of charge-carrier localization as a function of increasing edge-vacancy disorder is in agreement with other STB model studies (e.g., Refs. [10,19,21,22]). The fact that there is chargecarrier localization implies that, at longer device lengths, a conductance gap may also form as discussed in the following section.

\section{Variable device length and charge-carrier localization}

The $42.5 \%$ edge-vacancy concentrated system is chosen for detailed localization studies as previous sensitivity tests showed the 20-5-ZGNR to be within the localization regime, independent of the type of model investigated [Fig. 10(b)]. To begin this investigation, the noninteracting STB and ETB results are compared to determine whether the conductance at the Fermi energy drops below the $0.01 * e^{2} / h$ conduction- 


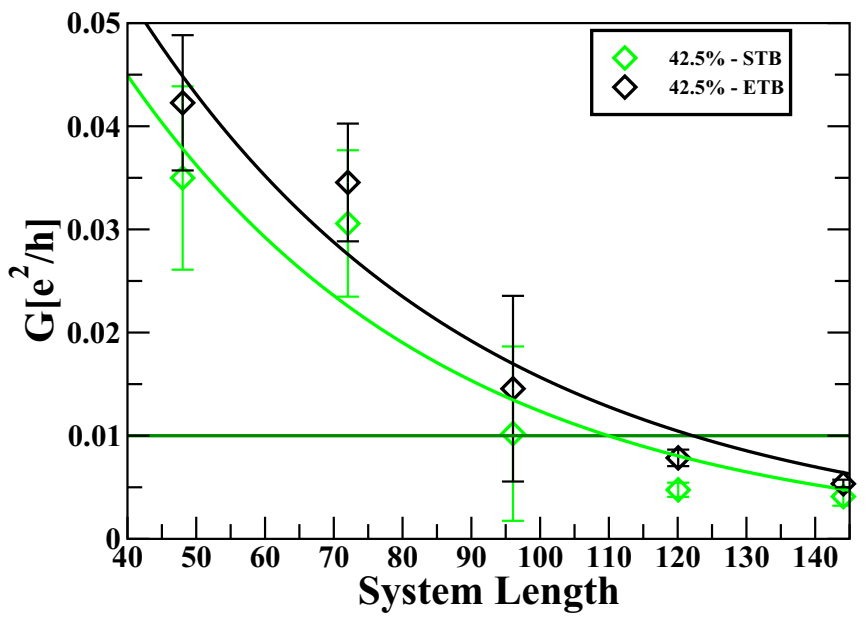

FIG. 11. Effect of the system length $(\AA)$ on the ensembleaveraged conductance $(\mathrm{G})$ measured at $E_{F}$ for random edgedisordered 5-ZGNRs at $42.5 \%$ edge-vacancy concentration. Green symbols represent the STB results and black symbols represent the ETB results. According to the zero conductance criterion $[5,20]$, a value of the conductance below the green line $\left(0.01 * e^{2} / h\right.$ threshold $)$ is equivalent to zero, thus indicating a conductance gap. Error bars correspond to the $S E$ uncertainties associated with these measurements.

gap criterion (for single spin), thus indicating the onset of a conductance gap as a function of increasing system length.

The ensemble-averaged conductance versus device length for the 5-ZGNR STB and ETB systems show that the ETB conductance remains larger than the STB results at all device lengths (Fig. 11). After fitting Eq. (11) to these results, localization lengths of $47 \AA$ and $49 \AA$ are determined for the STB and ETB systems, respectively, having maximum uncertainties of $\pm 3 \AA$. The fitting shows that the conductance at $E_{F}$ drops below the $0.01^{*} e^{2} / h$ threshold at $110 \AA$ for the STB system and $123 \AA$ for the ETB system, indicating the lengths at which a conduction gap forms. These observations are in agreement with previous trends, namely that the STB exhibits greater charge-carrier localization effects than the ETB model due to reduced kinetics.

In addition to these results, the criteria used by Cresti and Roche [21,22] is applied to determine the onset of the localization regime as a function of the system length for the $42.5 \%$ edge-vacancy-defected 5-ZGNRs. According to these criteria, the diffusion regime is well established when

$$
\frac{S E_{T}}{\langle T\rangle}<1
$$

whereas the localization regime is well defined at

$$
\frac{S E_{T}}{\langle T\rangle}>1
$$

and

$$
\frac{S E_{\ln (T)}}{\langle\ln (T)\rangle}<1,
$$

measured at $E_{F}$. Here, $\langle G\rangle=\left(e^{2} / h\right)\langle T\rangle$ links the ensemble average of $G$ with the ensemble average of the transmission $T$. The numerators in Eqs. (13)-(15) are the standard

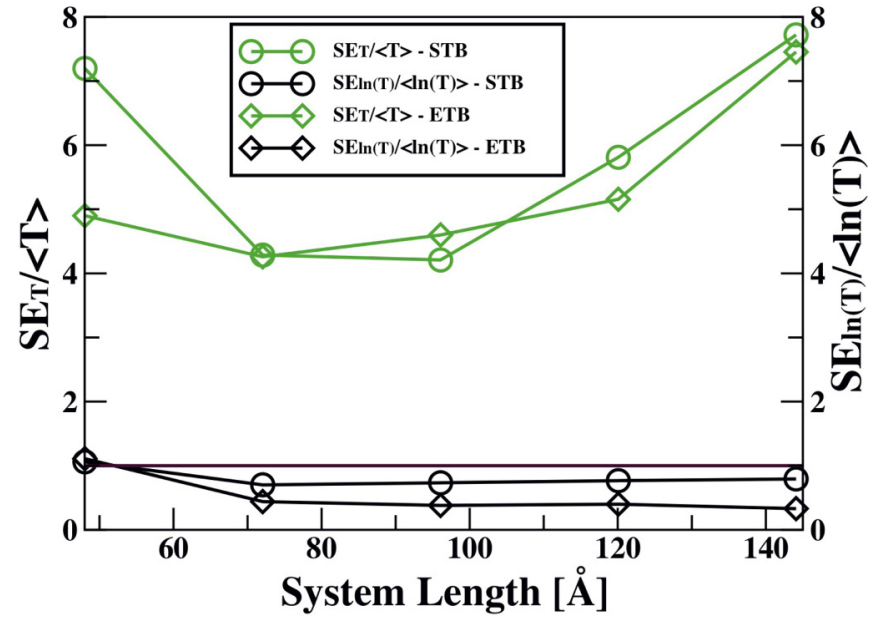

FIG. 12. Criteria used by Cresti and Roche (light green [Eq. (13)] and black [Eq. (14)]) as a function of increasing device length, measured in $\AA$, for random edge-vacancy disordered 5-ZGNRs with $42.5 \%$ edge-vacancy concentration. Circles correspond to the STB model and diamonds correspond to the ETB model. The horizontal line is where these criteria are established. Straight lines joining the data points are a guide to the eye only.

errors associated with the ensemble-averaged quantities in the denominators of these functions. Plotting these functions with respect to the device length shows that the 5-ZGNR with $42.5 \%$ edge-vacancy concentration is well within the localization regime for device lengths greater than $48.02 \AA$ for the 20-5-ZGNR, although this system is at the starting point for these criteria (Fig. 12).

To compare the gap properties of the STB, ETB, and GTB systems, the ensemble-averaged conductance gap is plotted as a function of the system length (Fig. 13). The error bars have been obtained by determining the gaps from the ensemble-averaged conductance results, plus and minus the

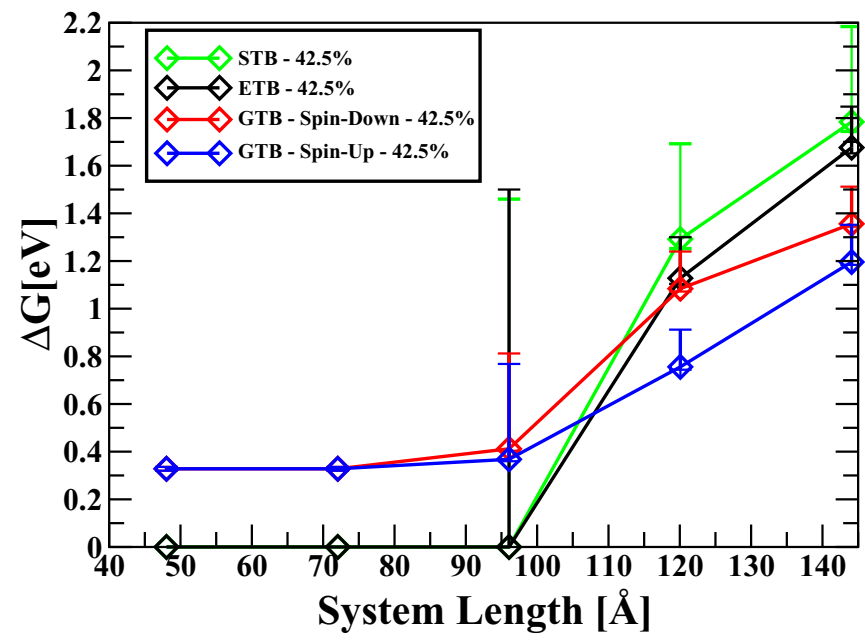

FIG. 13. Effect of device length on the ensemble-average conductance gap $\Delta G$ for random edge-disordered STB, ETB and GTB 5 -ZGNRs at $42.5 \%$ edge-vacancy concentration. The $0.01 * e^{2} / h$ conductance threshold [5,20] is assumed for determining zero conductance and in defining the formation of a conductance gap. 


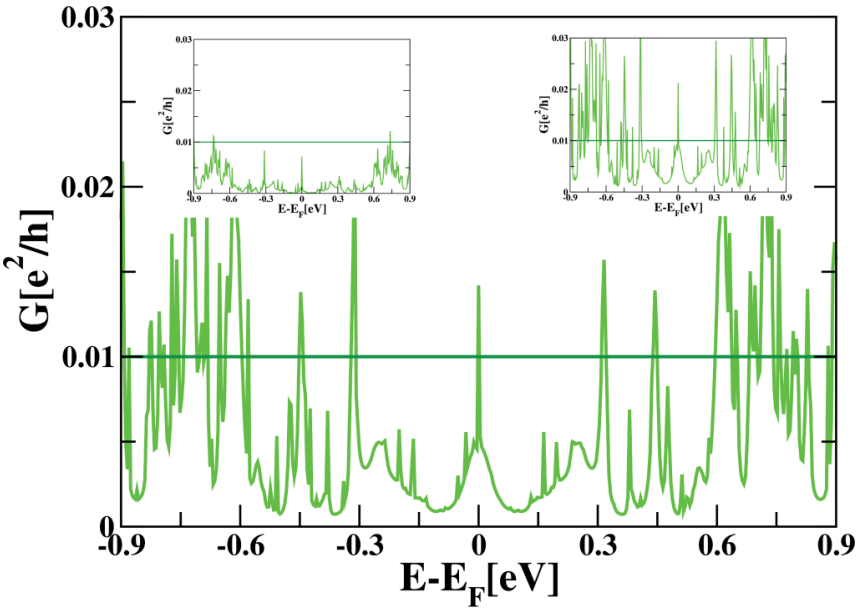

(a)

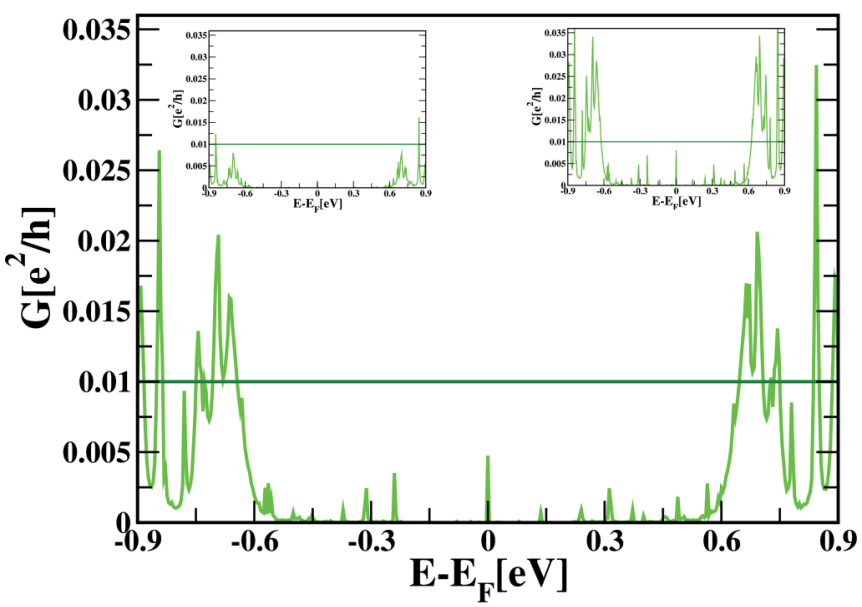

(b)

FIG. 14. Effect of the standard error $(S E)$ uncertainty on the coherent transport results. For the $L=96.1 \AA$ case shown in (a), the edge state appears above the $0.01 * e^{2} / h$ threshold criterion in the ensemble-averaged result (main graph) and in the $G-S E$ result (top-right inset) leading to zero gaps, whereas a finite gap occurs in the $G+S E$ solution (top-left inset). In the $L=120 \AA$ case shown in (b), the gap for $G-S E$ (top-inset right) is similar to the ensembleaveraged gap results (main graph), whereas the $G+S E$ case (topinset left) exhibits a larger gap.

associated $S E$ (i.e., from the $G+S E$ and $G-S E$ solutions), with the gaps defined using the $0.01 * e^{2} / h$ threshold criterion. The asymmetry in the gap uncertainties can be explained by example using the $L=96.1 \AA$ and $L=120 \AA$ STB results (Fig. 14). For the $L=96.1 \AA$ STB system [Fig. 14(a)], the edge state appears above the $0.01 * e^{2} / h$ threshold criterion in the ensemble-averaged result for $G$ (main figure) and in the $G-S E$ (top-right inset) results leading to zero conductance gaps, whereas a finite conductance gap occurs in the $G+S E$ solutions (top-left inset). In the $L=120 \AA$ case [Fig. 14(b)], the conductance gap in the $G-S E$ results (top-right inset) is similar in magnitude to the ensemble-averaged gap results (main figure), whereas the $G+S E$ case (top-left inset) exhibits a larger gap. The results at smaller system lengths (Fig. 13) show no uncertainties due to the stability of the edge states against disorder.
According to Fig. 13, the noninteracting STB and ETB models initially have a nonzero conductance gap, which opens for the 50-5-ZGNR $(L=120.1 \AA)$ with values of $1.29 \mathrm{eV}$ and $1.13 \mathrm{eV}$ for the ensemble-averaged STB and ETB results, respectively. For the 60-5-ZGNR $(L=144.1 \AA)$, the ensemble-averaged conduction gaps show further increase to $1.78 \mathrm{eV}$ for the STB model and $1.68 \mathrm{eV}$ for the ETB model. Compared to the zero-gap result for the noninteracting models, the GTB system has an intrinsic conductance gap of $0.328 \mathrm{eV}$ for the shorter 20- and 30-5-ZGNRs, increasing to $0.412 \mathrm{eV}$ for the spin-down and $0.368 \mathrm{eV}$ for the spin-up channels in the 40-5-ZGNR ( $L=96.1 \AA$ ), thus showing a breaking of the spin symmetry in the ensemble-averaged solutions. The GTB gap in the ensemble-averaged result further increases showing greater spin splitting for the longer 50-5-ZGNR (1.08 $\mathrm{eV}$ for spin-down and $0.756 \mathrm{eV}$ for spin-up) and 60-5-ZGNR (1.36 eV for spin-down and $1.20 \mathrm{eV}$ for spin-up).

The noninteracting STB and ETB systems require longer device lengths ( $>L=96.1 \AA$ ) for edge-vacancy perturbations to appear in the ensemble-averaged results (i.e., via the opening conduction gap) due to the edge states being more robust compared to those in the GTB interacting system. At these longer device lengths (50-5-ZGNR and 60-5-ZGNR), the noninteracting gaps become larger than the GTB gaps, which can be explained by the STB and ETB systems being more sensitive to the effects of edge-vacancy disorder. This difference can be explained by the Hubbard- $U$ in the GTB model introducing nontrivial competition between the kinetics, scattering (i.e., disorder-induced localization), and magnetic localization.

The culmination of these results indicates that disorderinduced localization is not the only gap-opening mechanism for edge-vacancy-defected ZGNRs that have persistent magnetic properties. Taking into account the uncertainties, key differences between the interacting and noninteracting systems remain, namely that the GTB shows spin-dependent gap properties not captured in the noninteracting models and that there are differences in the size of the gaps in these systems. The persistence of the magnetic state explained by Clar's rule in the ensemble-averaged results, coupled with the onset of spin-symmetry breaking due to random edge-vacancy defect perturbations, means that an interacting minimal model is ultimately required to describe these systems. An exception to this expectation may perhaps be in the limit of very high width and short length edge-vacancy-defected ZGNRs, and, in this respect, further work is required.

\section{CONCLUSION}

The stability of the Hubbard- $U$ conduction gap in the ensemble-averaged GTB results for random edge-vacancydefected 20-5-ZGNRs, even at high edge-vacancy concentrations, supports the use of the GTB model over simpler, noninteracting, model Hamiltonians in determining the properties of these systems. The effect of randomness and ensemble averaging was found to stabilize the spin polarization in these systems, resulting in persistent magnetism and spin-dependent properties. Such effects can be missed if systematic edgevacancy studies are performed. 
The effects of increasing device length were probed in 5-ZGNRs, with localization lengths determined as $47 \AA$ and $49 \AA( \pm 3 \AA)$ for the STB and ETB models, respectively. Disorder-induced localization requires longer device lengths ( $>96.1 \AA$ for the 40-5-ZGNR) to achieve the opening of a conduction gap in the ensemble-averaged noninteracting STB and ETB systems. The onset of the noninteracting conductance gap was investigated, with the ensemble-averaged STB and ETB gap results following the same increasing trend as the GTB model for device lengths $\geqslant 120 \AA$ A. Importantly, the ensemble-averaged GTB results had smaller conduction gaps and spin-dependent gap properties not captured by the noninteracting models. The culmination of these investigations leads to the conclusion that due to the nontrivial interplay between the Hubbard- $U$, kinetics and disorder, that an interacting model with ensemble averaging is required. These findings may be of interest when considering previous and future studies that use noninteracting models to calculate the properties of disordered ZGNRs.

\section{ACKNOWLEDGMENTS}

This work made use of the facilities of the N8 HPC Centre of Excellence, provided and funded by the N8 Consortium and EPSRC (United Kingdom), EP/K000225/1. We also acknowledge the University of York's award of a dedicated cluster (Nano) ascribed to Y.H.'s research group for theoretical graphene research. An EPSRC (United Kingdom), EP/P505798/1, PhD studentship was awarded to Y.H. from the Department of Physics for J.P.C.B. All data created during this research are available by request from the University of York Data Catalogue http://dx.doi.org/10.15124/8b64bbc8162d-4550-bcf2-b248e0ad4fc5.
[1] C. Tao, L. Jiao, O. V. Yazyev, Y.-C. Chen, J. Feng, X. Zhang, R. B. Capaz, J. M. Tour, A. Zettl, S. G. Louie, H. Dai, and M. F. Crommie, Nat. Phys. 7, 616 (2011).

[2] K. A. Ritter and J. W. Lyding, Nat. Mater. 8, 235 (2009).

[3] F. Molitor, C. Stampfer, J. Güttinger, A. Jacobsen, T. Ihn, and K. Ensslin, Semicond. Sci. Technol. 25, 034002 (2010).

[4] P. Gallagher, K. Todd, and D. Goldhaber-Gordon, Phys. Rev. B 81, 115409 (2010).

[5] M. Y. Han, B. Özyilmaz, Y. Zhang, and P. Kim, Phys. Rev. Lett. 98, 206805 (2007).

[6] Y.-W. Son, M. L. Cohen, and S. G. Louie, Phys. Rev. Lett. 97, 216803 (2006).

[7] M. Y. Han, J. C. Brant, and P. Kim, Phys. Rev. Lett. 104, 056801 (2010).

[8] C. Stampfer, J. Güttinger, S. Hellmüller, F. Molitor, K. Ensslin, and T. Ihn, Phys. Rev. Lett. 102, 056403 (2009).

[9] Z. Chen, Y.-M. Lin, M. J. Rooks, and P. Avouris, Physica E 40, 228 (2007).

[10] X. Li, X. Wang, L. Zhang, S. Lee, and H. Dai, Science 319, 1229 (2008).

[11] X. Li, G. Zhang, X. Bai, X. Sun, X. Wang, E. Wang, and H. Dai, Nat. Nanotechnol. 3, 538 (2008).

[12] D. V. Kosynkin, A. L. Higginbotham, A. Sinitskii, J. R. Lomeda, A. Dimiev, B. K. Price, and J. M. Tour, Nature (London) 458, 872 (2009).

[13] L. Jiao, L. Zhang, X. Wang, G. Diankov, and H. Dai, Nature (London) 458, 877 (2009).

[14] J. Feng, W. Li, X. Qian, J. Qi, L. Qi, and J. Li, Nanoscale 4, 4883 (2012).

[15] B. Huang, F. Liu, J. Wu, B.-L. Gu, and W. Duan, Phys. Rev. B 77, 153411 (2008).

[16] D. A. Areshkin, D. Gunlycke, and C. T. White, Nano Lett. 7, 204 (2007).

[17] D. Gunlycke, D. A. Areshkin, and C. T. White, Appl. Phys. Lett. 90, 142104 (2007).

[18] A. Cresti, N. Nemec, B. Biel, G. Niebler, F. Triozon, G. Cuniberti, and S. Roche, Nano Res. 1, 361 (2008).

[19] E. R. Mucciolo, A. H. Castro Neto, and C. H. Lewenkopf, Phys. Rev. B 79, 075407 (2009).

[20] M. Evaldsson, I. V. Zozoulenko, H. Xu, and T. Heinzel, Phys. Rev. B 78, 161407 (2008).
[21] A. Cresti and S. Roche, Phys. Rev. B 79, 233404 (2009).

[22] A. Cresti and S. Roche, New J. Phys. 11, 095004 (2009).

[23] T. C. Li and S.-P. Lu, Phys. Rev. B 77, 085408 (2008).

[24] I. Martin and Y. M. Blanter, Phys. Rev. B 79, 235132 (2009).

[25] D. Querlioz, Y. Apertet, A. Valentin, K. Huet, A. Bournel, S. Galdin-Retailleau, and P. Dollfus, Appl. Phys. Lett. 92, 042108 (2008).

[26] P. W. Anderson, Phys. Rev. 109, 1492 (1958).

[27] C. T. White, J. Li, D. Gunlycke, and J. W. Mintmire, Nano Lett. 7, 825 (2007)

[28] Y. Hancock, A. Uppstu, K. Saloriutta, A. Harju, and M. J. Puska, Phys. Rev. B 81, 245402 (2010).

[29] Y. Hancock, K. Saloriutta, A. Uppstu, A. Harju, and M. J. Puska, J. Low Temp. Phys. 153, 393 (2008).

[30] J. Hubbard, Proc. R. Soc. Lond. A 276, 238 (1963).

[31] Y. Hancock and A. E. Smith, Physica E 18, 383 (2003).

[32] D. E. Knuth, The Art of Computer Programming, (3rd ed.): Seminumerical Algorithms Vol. 2 (Addison-Wesley Longman Publishing Co., Reading, MA, 1997).

[33] Z. Liu, K. Suenaga, P. J. F. Harris, and S. Iijima, Phys. Rev. Lett. 102, 015501 (2009).

[34] S. Datta, Electronic Transport in Mesoscopic Systems (Cambridge University Press, Cambridge, U.K., 1997).

[35] F. Guinea, C. Tejedor, F. Flores, and E. Louis, Phys. Rev. B 28, 4397 (1983).

[36] F. Muñoz-Rojas, D. Jacob, J. Fernández-Rossier, and J. J. Palacios, Phys. Rev. B 74, 195417 (2006).

[37] E. H. Lieb, Phys. Rev. Lett. 62, 1201 (1989).

[38] E. Clar, Polycyclic Hydrocarbons (Academic Press, London, 1964).

[39] E. Clar, The Aromatic Sextet (Wiley, London, 1972).

[40] T. Wassmann, A. P. Seitsonen, A. M. Saitta, M. Lazzeri, and F. Mauri, J. Am. Chem. Soc. 132, 3440 (2010).

[41] O. V. Yazyev, Rep. Prog. Phys. 73, 056501 (2010).

[42] D. J. Thouless, J. Phys. C: Solid State Phys. 6, L49 (1973).

[43] R. Landauer, Philos. Mag. 21, 863 (1970).

[44] P. W. Anderson, D. J. Thouless, E. Abrahams, and D. S. Fisher, Phys. Rev. B 22, 3519 (1980).

[45] R. Johnston and H. Kunz, J. Phys. C: Solid State Phys. 16, 3895 (1983). 